\title{
TV White Spaces in Public Libraries: A Primer
}

Kristen Radsliff Rebmann, Emmanuel Edward Te, and Donald Means

\begin{abstract}
TV White Space (TVWS) represents one new wireless communication technology that has the potential to improve internet access and inclusion. This primer describes TVWS technology as a viable, long-term access solution for the benefit of public libraries and their communities, especially for underserved populations. Discussion focuses first on providing a brief overview of the digital divide and the emerging role of public libraries as internet access providers. Next, a basic description of TVWS and its features is provided, focusing on key aspects of the technology relevant to libraries as community anchor institutions. Several TVWS implementations are described with discussion of TVWS implementations in several public libraries. Finally, consideration is given to first steps that library organizations must take when contemplating new TVWS implementations supportive of WiFi applications and crisis response planning .
\end{abstract}

\section{INTRODUCTION}

Tens of millions of people rely wholly or in part on libraries to provide access to the Internet. Many lack access to the Federal Communications Commission (FCC) recommended standard of 25 Mbps (megabits per second) download speed and 3 Mbps upload speed. ${ }^{1}$ Though the FCC reclassified high-speed Internet as a public utility under Title II of the Telecommunications Act to ensure that broadband networks are "fast, fair, and open" in $2015,{ }^{2}$ the "digital divide" still remains. One in four community members does not have access to the Internet at home. Accounting for age and education level, households with the lowest median income households have service adoption rates of around 50\%, compared to those with higher incomes, with rates of 80 to $90 \%{ }^{3}$ A recent Pew Research Center survey on home broadband adoption found that $43 \%$ of those surveyed reported cost being their main reason for non-adoption. ${ }^{4}$ Individuals with low quality or no access are more likely to be digitally disadvantaged, tend to use library computers more frequently, and are less equipped to interact and compete economically as more services and application processes move online. ${ }^{5}$

Kristen Radsliff Rebmann (Kristen.rebmann@sjsu.edu) is Associate Professor, San Jose State University School of Information, San Jose, CA. Emmanuel Edward Te (emmanueledward.te@sjsu.edu) is a graduate student, San Jose State University School of Information, San Jose, CA. Donald Means (don@digitalvillage.com) is co-founder and principal of Digital Village Associates, Sausalito, CA. 
This article highlights TV White Space (TVWS), a new wireless communication technology with the potential to assist libraries in addressing digital access and inclusion issues. This primer provides first a brief overview of the digital divide and the emerging role of public libraries as internet access providers, highlighting the need for cost-efficient, technological solutions. We go further to provide a basic description of TVWS and its features, focusing on key aspects of the technology relevant to libraries as community anchor institutions. Several TVWS implementations are described with discussion of how TVWS was set up in several public libraries. Finally, we extend consideration to first steps library organizations must consider when contemplating new implementations including everyday applications and crisis response planning.

\section{Digital Access and Inclusion}

The term "digital divide" describes the gap between people who can easily access and use technology and the internet, and those who cannot. ${ }^{6}$ As Kinney observes, "there has not been one single digital divide, but rather a series of divides that attend each new technology." 7 Digital divides are exacerbated by various factors including: socioeconomic status, education, geography, age, ability, language, and especially availability and quality. ${ }^{8}$ In recent years, the language describing this issue has changed, but the inequalities stay consistent and widen among different dimensions with each emerging technology. The most recent public policy term "digital inclusion" promotes digital literacy efforts for unserved and underserved populations. ${ }^{9}$ The progression from the term "digital divide" to "digital inclusion" represents a shift in focus from issues of access exclusively toward contexts and quality of participation and usage. Along these lines, the language of digital inclusion reframes the issue by making visible that simply focusing on internet access can obscure the fact that divides associated with quality and effectiveness remain. ${ }^{10}$

In response to the digital divide, public libraries have become the "unofficial" providers of internet access, stemming from libraries' access to broadband infrastructure, maintenance of publiclyavailable computers, and services providing assistance and training. ${ }^{11} \mathrm{~A}$ Pew Research Center survey on perceptions of libraries found that most respondents reported viewing public libraries as important parts of their communities, providing resources and assisting in decisions regarding what information to trust. ${ }^{12}$ However, many public libraries are facing an "infrastructure plateau" of internet access due to few computer workstations and slower broadband connection speeds that can support a growing number of users, ${ }^{13}$ on top of insufficient funding, physical space, and staffing. ${ }^{14}$ Previous surveys show that although public libraries are connected to the internet and provide public access workstations and wireless access, nearly $50 \%$ of public libraries only offer wireless access that shares the same bandwidth as their workstations. ${ }^{15}$ This increased usage strains existing network connections and infrastructure, resulting in slower connections for everyone connected to the public library's network. Many public libraries cannot accommodate more workstations, support the power requirements of both workstations and patrons' laptops, and afford workstation upgrades and bandwidth increases to move past their insufficient connectivity speeds. Libraries often lack the IT skills, time, and funds to upgrade their 
infrastructure. ${ }^{16}$ Typical wireless access via Wi-Fi is relegated to distances within library buildings, which may extend to exterior spaces and is available only during operating hours.

Despite these challenges, public libraries continually provide access and "at-the-point-of-need" training and support for their patrons, especially for those who do not have easy access to the internet and computers. ${ }^{17}$ Subsidized by federal funding, libraries represent key access providers and technology trainers for the public without internet access. ${ }^{18}$ The FCC classifies libraries as "community anchor institutions" (CAIs), organizations that "facilitate greater use of broadband by vulnerable populations, including low-income, the unemployed, and the aged." 19 Recent surveys show that users have a positive view of libraries, providing opportunities to spend time in a safe space, pursue learning, and promote a sense of community. Librarians offer internet skills training programs more often than other community organizations though (at around 75\% of the time) training occurs informally. ${ }^{20}$ In particular, $29 \%$ of respondents to a library use survey reported going to libraries to use computers, the internet, or the Wi-Fi network; 7\% have also reported using libraries' Wi-Fi signals outside when libraries are closed. ${ }^{21}$ The majority of these users are more likely to be young, black, female, and lower income, utilizing library technology resources for school or work (61\%), checking email or sending texts (53\%), finding health information (38\%), and taking online courses or completing certifications $(26 \%){ }^{22}$

Public libraries are already exploring creative approaches to providing internet access for these underserved communities. The mobile hotspot lending program in public library systems in New York City and Kansas City are just two examples. ${ }^{23}$ Yet libraries must do more by supporting innovation and providing leadership by partnering with other community organizations and their stakeholders to enhance resilience in addressing access and inclusion. The emergence of TVWS wireless technology presents an opportunity for libraries to explore expanding the reach of their wireless signals beyond library buildings and extend 24/7 library Wi-Fi availability to community spaces such as subsidized housing, schools, clinics, parks, senior centers, and museums.

\section{TVWS Basics}

TV Whitespace (TVWS) refers to the unoccupied portions of spectrum in the VHF/UHF terrestrial television frequency bands. ${ }^{24}$ Television broadcast frequency allocations traditionally assumed that TV station transmissions operating at high power needed wide spectrum separation to prevent interference between broadcasting channels, which led to the specific spectrum allocation of these frequency "guard bands." 25 Research discovered that low-power devices can operate within these spaces, which led the Federal Communications Commission (FCC) to field test TVWS applications to wireless communications and (ultimately) promote TVWS neutrality. ${ }^{26}$ In 2015 , the Federal Communications Commission (FCC) made a portion of these very valuable TVWS bands of spectrum available for open, shared public use, like Wi-Fi. Yet, unlike Wi-Fi, with a reach measured in 10s of meters, the range of TVWS is measured in 100s or even 1000s of meters. TVWS has good propagation characteristics, which makes it an extremely valuable license-exempt radio spectrum. ${ }^{27}$ It is a relatively stable frequency that does not change over time, allowing for 
spectrum availability estimates to remain reliable and valid, which in turn promotes its various applications. ${ }^{28}$ Radio spectrum is considered a "common heritage of humanity," 29 as radio waves "do not respect national borders." 30 The FCC recently made a portion of these TVWS bands of spectrum available for open, shared public use. ${ }^{31}$

TVWS availability and application are contextual and dependent on many key factors. Availability is influenced by frequency (the idle channels purposely planned in TV bands, varying across regions), deployment (the height and location of the TVWS transmit antenna and its installation sites in relation to nearby surrounding TV broadcasting reception), space and distance (geographical areas outside the current planned TV coverage, including no present broadcasting signals), and time (off-air availability of licensed broadcasting transmitters during specific periods of time, subject to change by the broadcaster). ${ }^{32}$ As TVWS existed as fragmented "safety margins" between broadcast services, TVWS is typically more abundant in rural areas that have less broadcast coverage and in larger contiguous blocks rather than in highly dense urban areas. ${ }^{33}$

Assigned spectrum is not always used efficiently and effectively by licensees, and exclusive or nonexclusive sharing can alleviate pressure on these resources. ${ }^{34}$ This "spectrum crunch" of the inefficient use of scarce spectrum resources can be alleviated with dynamic spectrum access (DSA) and spectrum sharing. TVWS availability is small where digital television has been deployed, with the potentials for aggregate interference (from TVWS users in relation to primary TV service) and self-interference (within the TVWS network), which may lead to a "mismatch situation" where there is high demand for bandwidth but very low TVWS bandwidth supply. ${ }^{35}$ As most spectrum frequencies have been organized through some form of exclusive access in which only the licensee can use the specific spectrum, technologies such as cognitive radios can enable new modes of spectrum access, supporting autonomous, self-configuring, self-planning networks which rely on up-to-date TVWS availability databases.

The limited distribution (in many areas) of basic broadband infrastructure and relatively high cost of access often prevents individuals with lower incomes from participating in the digital revolution of information access and its opportunities. ${ }^{36}$ Despite these challenges to broadband availability, TVWS excels in areas with low broadband coverage. Rural regions possess greater frequency availability due to lower density of spectrum licensing. In comparison to other frequencies operating higher up on the spectrum band, TVWS does not require direct line-of-sight between devices for operation, and has lower deployment costs. Equipment market costs are comparable to Wi-Fi equipment currently on the market. ${ }^{37}$

Importantly, TVWS can address access and inclusion by having relatively low start-up costs and no ongoing services fees. As a public resource, it can work with existing services to create new, potentially mobile connections to the internet that ensure the continuation of vital services in the event of service interruptions. ${ }^{38}$ In urban areas with fewer channels available, new efficient spectrum sharing policies will be necessary. Assigned spectrum is not always used efficiently and effectively by licensees, and exclusive or non-exclusive sharing or "recycling" of bands for more 
effective spectrum use by multiple parties with changing spectrum needs can alleviate pressure on these resources. ${ }^{39}$

\section{TVWS for Public Libraries}

TVWS is a viable medium for applications from internet access, content distribution within a given location, tracking (people, animals, and assets), task automation, and public safety and security, ${ }^{40}$ as well as remote patient monitoring and other telemedicine applications. ${ }^{41}$ TVWS complements existing networks that use other parts of the spectrum for access points, mobile communications, and home media devices. ${ }^{42}$ Analyses of a recent digital inclusion survey suggest that technology upgrades can have significant impact on the ability of libraries to expand programs and services. ${ }^{43}$ As community anchor institutions (CAIs), public libraries can use TVWS systems to expand and improve access to their services for their users, especially for underserved populations.

Library-led collaborations to deploy TVWS networks in other CAIs and public spaces have numerous benefits. In conjunction with building-centered Wi-Fi, TVWS can redistribute network users from congested library spaces to other community sites, thereby distributing network usage across the community. From an existing broadband connection, libraries can extend their networks of internet access strategically across their communities. Yet, unlike networks which solely use limited-range Wi-Fi, far-reaching TVWS can improve the coverage and inclusion of patrons in accessing library programs, services and the broader internet. ${ }^{4}$ The portability of the access points allows libraries to extend their reach by providing wireless connections in the shortterm, for cultural or civic events like fairs, markets, or concerts, and in the long-term, for use at popular public areas.

Recent TVWS pilot installations have proven to be very stable in Kansas, Colorado, Mississippi, and Delaware. Manhattan Public Library (Kansas)'s TVWS project began in fall 2013. Though there were a few delays in the installation and testing process, the TVWS equipment was successfully implemented and welcomed by the community in early 2014. IT staff report that their remote locations have shown that this library service fills a community need, especially for underserved populations. ${ }^{45}$ Delta County Libraries (Colorado) are conducting trials with two public hotspots to support "Guest" access and potentially provide library patrons with more bandwidth access. ${ }^{46}$ TVWS implementations in the Pascagoula School District (Mississippi) ${ }^{47}$ and Delaware Public Libraries ${ }^{48}$ show successful initial pilot usage in providing wireless internet service directly to community-distributed access points. Though there are contextual differences across these sites, the strength of public libraries as CAIs providing internet access via TVWS systems is evident and promising.

\section{First Steps}

Any library can take the initiative in setting up a TVWS network on its own. The first step is to assess availability of spectrum in the library's geographic location. Access to TVWS frequencies is free and requires no subscription fees other than the initial equipment investment. Public 
databases of TVWS availability are easily accessible and have been tested by the FCC since 2011;49 Google also has posted its own spectrum database as well. ${ }^{50}$ From this setup, the library gains access to public TVWS frequencies by which they can broadcast and receive internet connections from paired TVWS-enabled remote hotspots. Once it is determined that there is available spectrum/channels in the desired area, libraries can then explore how their current broadband and wireless connections might be expanded to include several community spaces where internet access is needed. Next, the library works with a TVWS equipment supplier to design and install a TVWS network consisting of a base station that is integrated with their wired connection to the internet. Finally, the library places TVWS-enabled remote hotspots in (previously identified) community-based spaces where Wi-Fi access is needed by underserved populations. Given a high quality backhaul (i.e., fiber optic cable high speed connection), TVWS can spread that signal and provide access from the library, which is able to propagate and penetrate multiple barriers and geographical features with a signal up to 10 times stronger than current Wi-Fi. Depending on the context (geographical features, TVWS availabilities, etc.), hotspots can be installed up to six miles (10 km) away and do not require line-of-sight between the base station and hotspots. This ability is superior to current Wi-Fi networks that only cover patrons in the immediate vicinity of the library. These TVWS remote hotspots also can be easily (and strategically) moved to support occasional community needs (such as neighborhood-wide or city events) or in response to crisis situations.

\section{TVWS, Libraries, and Emergency Response}

Public libraries provide leadership as "ready access point, first choice, first refuge, and last resort" for community services in everyday matters and in emergencies. ${ }^{51}$ They have assisted residents in relief efforts during Hurricanes Katrina and Rita, and other natural and man-made disasters. ${ }^{52}$

...the provision of access to computers and the internet was a wholly unique and immeasurably important role for public libraries... The infrastructure can be a tremendous asset in times of emergencies, and should be incorporated into community plans. ${ }^{53}$

They have likewise provided immediate and long-term assistance to communities and aid workers, providing physical space for recovery operations for emergency agencies, communication technologies, and emotional support for the community. In previous library internet usage surveys, nearly one-third of libraries reported that their computers and internet services would be used by the public in emergencies to access relief services and benefits. ${ }^{54}$ Such activities include finding and communicating with family and friends, completing online FEMA forms and insurance claims, and checking news sites regarding information of their affected homes. ${ }^{55}$

Yet, despite the admirable and successful efforts of many public libraries, their infrastructures are not always built to meet the increased demand of user needs and e-government services in emergency contexts. ${ }^{56}$ Jaeger, Shneiderman, Fleischmann, Preece, Qu, and Wu propose the concept of community response grids (CRGs), which utilize the internet and mobile communications devices so that emergency responders and residents in a disaster area can 
communicate and coordinate accurate, appropriate responses. ${ }^{57}$ This concept relies on social networks, both in person and online, to enable residents and emergency responders to work together in a multi-directional communication scheme. CRGs provide residents tailored, localized information and a means to report pertinent disaster related information to emergency responders, who in turn can synthesize and analyze submitted information and act accordingly. ${ }^{58}$

Due to their existing role as community anchor institutions (CAIs), public libraries are uniquely positioned for CRG involvement. Libraries can assist in facilitation of internet access with portable TVWS network connection points. By virtue of their portability, TVWS hotspots can provide essential digital access in times of crisis by moving along with their affected populations. Emergency operations and communications in a crisis occur throughout networks comprised of various technologies. Information management before, during, and after a disaster affects how well a crisis is managed. ${ }^{59}$ Broadband internet can be one access route in the event that phone and radio transmissions are affected, and vice versa, as part of a "mixed media approach" to get messages to those that need it in an emergency. ${ }^{60}$ Yet one must remember that internet communications are double-edged: the internet provides relevant material on demand and near instant sharing and collaborating, but these very features can compound a crisis with misinformation. ${ }^{61}$ Despite these concerns, the potential of the integration of wireless devices and other technologies into a multi-technology, collaborative response system can solve the problem of existing communication structures that lack coordination and quality control.62 The proliferation of smartphones, laptops, and other portable wireless devices makes such technology ideal for emergency communications, especially in how users' familiarity with their own devices will help them navigate CRG communications while under stress. ${ }^{63}$

\section{CONCLUSION}

Supporting internet access and inclusion in public libraries and having equal, affordable, and available access to information is a necessary component to bridging the digital divide.

Technology has become "an irreducible component of modern life, and its presence and use has significant impact on an individuals' ability to fully engage in society." 64 As Cohron argues, this principle represents more than providing people with internet access: it is about "leveling the playing field in regards to information diffusion. The internet is such a prominent utility in peoples' lives that we, as a society, cannot afford for citizens to go without." 65 Broadband access is the first step; digital literacy training is also a necessity. Access alone is not enough to ensure quality and effective use, however, as the digital divide is representative of broader social inequalities that computer and internet access cannot fully remedy. ${ }^{66}$ This is a complex problem that requires a multi-faceted solution. As Kinney states, "the digital divide is a moving target, and new divides open up with new technologies. Libraries help bridge some inequities more than others, and substantial disparities exist among library systems." 67 Internet access also becomes a necessity when the internet is to play a role in emergency communications. ${ }^{68}$ 
It is problematic to suggest that public libraries can be simultaneously promoted as the solution to digital divide issues while facing cuts to funding. Policy makers, community advocates, and the community members themselves are stakeholders in the success of their communities, and must also take responsibility for access and inclusion via public libraries. ${ }^{69}$ As public agencies automate to increase equality and save money, they exacerbate digital divides by excluding those without access. Suggesting that community members simply visit the library to ensure access to public services places additional pressure on libraries, yet these efforts may go unsupported and unacknowledged.

Public libraries are already valuable community access points to resources especially in emergencies, though many suffer from a lack of concerted disaster planning. Along similar lines, many libraries are ill-equipped to accommodate the bandwidth needs of growing and oftentimes sparsely connected populations. As communications and government services move increasingly online, it becomes imperative to build strong cost-effective information infrastructures. TVWS connections can arguably help in breaking down the barriers that challenge ubiquitous access and inclusion. TVWS-enabled remote access points in daily use around communities are ideally situated to provide everyday Wi-Fi and for rapid redeployment to damaged areas (as pop-up hotspots) to provide essential communication and information resources in times of crisis. In short, TVWS can augment the technological infrastructure of public libraries toward further developing their roles as CAIs and leaders serve their communities well into the future.

\section{REFERENCES}

1. Wireline Competition Bureau, "2016 Broadband Progress Report," Federal Communications Commission, January 29, 2016, https://www.fcc.gov/reports-research/reports/broadbandprogress-reports/2016-broadband-progress-report.

2. Office of Chairman Wheeler, "FCC Adopts Strong, Sustainable Rules to Protect the Open Internet," Federal Communications Commission, February 26, 2015, https://apps.fcc.gov/edocs public/attachmatch/DOC-332260A1.pdf.

3. "Here's What the Digital Divide Looks Like in the United States," The White House ${ }_{2}$ July 15, 2015, https://www.whitehouse.gov/share/heres-what-digital-divide-looks-united-states.

4. John B. Horrigan and Maeve Duggan, "Home Broadband 2015," Pew Research Center, December 21, 2015, http://www.pewInternet.org/files/2015/12/Broadband-adoptionfull.pdf. This $43 \%$ is further divided between $33 \%$ reporting the monthly subscription cost as their main reason, while the other $10 \%$ report the expensive cost of a computer as their reason for non-adoption.

5. Bo Kinney, "The Internet, Public Libraries, and the Digital Divide," Public Library Quarterly 29, no. 2 (2010): 104-161, https://doi.org/10.1080/01616841003779718. 
6. Madalyn Cohron, "The Continuing Digital Divide in the United States," The Serials Librarian 69, no. 1 (2015): 77-86, https://doi.org/10.1080/0361526X.2015.1036195.

7. Kinney, “The Internet, Public Libraries, and the Digital Divide."

8. Paul T. Jaeger, John Carlo Bertot, Kim M. Thompson, Sarah M. Katz, and Elizabeth J. DeCoster, "The Intersection of Public Policy and Public Access: Digital Divides, Digital Literacy, Digital Inclusion, and Public Libraries," Public Library Quarterly 31, no.1 (2012): 1-20, https://doi.org/10.1080/01616846.2012.654728.

9. Brian Real, John Carlo Bertot, and Paul T. Jaeger, "Rural Public Libraries and Digital Inclusion: Issues and Challenges," Information Technology and Libraries 33, no. 1 (2014): 6-24, https://doi.org/10.6017/ital.v33i1.5141.

10. Jaeger et al., "The Intersection of Public Policy and Public Access.”

11. John Carlo Bertot, Paul T. Jaeger, Lesley A. Langa, Charles R. McClure, "Public access computing and Internet access in public libraries: The role of public libraries in e-government and emergency situations," First Monday 11, no. 9 (2006), https://doi.org/10.5210/fm.v11i9.1392.

12. John. B Horrigan, “Libraries 2016,” Pew Research Center, September 9. 2016, http://www.pewinternet.org/2016/09/09/libraries-2016/.

13. Real et al., "Rural Public Libraries and Digital Inclusion.”

14. John Carlo Bertot, Charles R. McClure, and Paul T. Jaeger, "The Impacts of Free Public Internet Access on Public Library Patrons and Communities," Library Quarterly 78, no.3 (2008): 285301, https://doi.org/10.1086/588445.

15. Charles R. McClure, Paul T. Jaeger, John Carlo Bertot, "The Looming Infrastructure Plateau? Space, Funding, Connection Speed, and the Ability of Public Libraries to meet the Demand for Free Internet Access," First Monday 12, no. 12 (2007):

https://doi.org/10.5210/fm.v12i12.2017.

16. Ibid.

17. Bertot et al., "Public access computing and Internet access in public libraries."

18. Ibid.; Jaeger et al., "The Intersection of Public Policy and Public Access."

19. Wireline Competition Bureau, "WCB Cost Model Virtual Workshop 2012 - Community Anchor Institutions," Federal Communications Commission, June 1, 2012, https://www.fcc.gov/newsevents/blog/2012/06/01/wcb-cost-model-virtual-workshop-2012-community-anchorinstitutions.

20. Jennifer Koerber, "ALA and iPAC Analyze Digital Inclusion Survey," Library Journal 141, no. 1 (2016): 24-26.

21. Horrigan, “Libraries 2016.” 
22. Ibid.

23. Timothy Inklebarger, "Bridging the tech gap," American Libraries, September 11, 2015, https://americanlibrariesmagazine.org/2015/09/11/bridging-tech-gap-wi-fi-lending.

24. Andrew Stirling, "White spaces - the new Wi-Fi?," International Journal of Digital Television 1, no. 1 (2010): 69-83, https://doi.org/10.1386/jdtv.1.1.69/1; Cristian Gomez, "TV White Spaces: Managing Spaces or Better Managing Inefficiencies?," in TV White Spaces A Pragmatic Approach, eds. Ermanno Pietrosemoli and Marco Zennaro (Trieste: Abdus Salam International Centre for Theoretical Physics T/ICT4D Lab, 2013), 67-77.

25. Steve Song, "Spectrum and Development," in TV White Spaces A Pragmatic Approach, eds. Ermanno Pietrosemoli and Marco Zennaro (Trieste: Abdus Salam International Centre for Theoretical Physics T/ICT4D Lab, 2013), 35-40.

26. Robert Horvitz, "Geo-Database Management of White Space vs. Open Spectrum," in TV White Spaces A Pragmatic Approach, eds. Ermanno Pietrosemoli and Marco Zennaro (Trieste: Abdus Salam International Centre for Theoretical Physics T/ICT4D Lab, 2013), 7-17.

27. Julie Knapp, "FCC Announces Public Testing of First Television White Spaces Database," Federal Communications Commission, September 14, 2011, https://www.fcc.gov/newsevents/blog/2011/09/14/fcc-announces-public-testing-first-television-white-spacesdatabase.

28. Horvitz, "Geo-Database Management of White Space vs. Open Spectrum."

29. Ryszard Strużak and Dariusz Więcek, "Regulatory Issues for TV White Spaces," in TV White Spaces A Pragmatic Approach, eds. Ermanno Pietrosemoli and Marco Zennaro (Trieste: Abdus Salam International Centre for Theoretical Physics T/ICT4D Lab, 2013), 19-34.

30. Horvitz, "Geo-Database Management of White Space vs. Open Spectrum," 8.

31. Engineering \& Technology Bureau, "FCC Adopts Rules For Unlicensed Services In TV And 600 MHz Bands," Federal Communications Commission, August 11, 2015, https://apps.fcc.gov/edocs_public/attachmatch/FCC-15-99A1_Rcd.pdf.

32. Gomez, “TV White Spaces: Managing Spaces or Better Managing Inefficiencies?," 68.

33. Stirling, "White spaces - the new Wi-Fi?."

34. Linda E. Doyle, "Cognitive Radio and Africa," in TV White Spaces A Pragmatic Approach, eds. Ermanno Pietrosemoli and Marco Zennaro (Trieste: Abdus Salam International Centre for Theoretical Physics T/ICT4D Lab, 2013), 109-119.

35. Gomez, "TV White Spaces: Managing Spaces or Better Managing Inefficiencies?," 72.

36. Mike Jensen, "The role of TV White Spaces and Dynamic Spectrum in helping to improve Internet access in Africa and other Developing Regions," in TV White Spaces A Pragmatic 
Approach, eds. Ermanno Pietrosemoli and Marco Zennaro (Trieste: Abdus Salam International Centre for Theoretical Physics T/ICT4D Lab, 2013), 83-89.

37. Song, "Spectrum and Development."

38. Ibid.

39. Doyle, “Cognitive Radio and Africa,” 113.

40. Stirling, "White spaces - the new Wi-Fi?."

41. Afton Chavez, Ryan Littman-Quinn, Kagiso Ndlovu, and Carrie L Kovarik, "Using TV white space spectrum to practice telemedicine: A promising technology to enhance broadband Internet connectivity within healthcare facilities in rural regions of developing countries," Journal of Telemedicine and Telecare 22, no. 4 (2015): 260-263, https://doi.org/10.1177/1357633X15595324.

42. Stirling, "White spaces - the new Wi-Fi?."

43. Koerber, "ALA and iPAC Analyze Digital Inclusion Survey."

44. Chavez et al., "Using TV white space spectrum to practice telemedicine."

45. Kerry Ingersoll, June 22, 2015, Google+ comment to the Gigabit Libraries Network, https://plus.google.com/107631107756352079114/posts/L4Y8ci8sG5Y.

46. Delta County Libraries, "Super Wi-Fi Pilot,” accessed November 1, 2016, http://www.deltalibraries.org/super-wi-fi-pilot/.

47. Pascagoula TV White Spaces Facebook group, accessed November 1, 2016, https://www.facebook.com/PSDTVWS/.

48. “Delaware Libraries White Space Pilot Update, January 2015," accessed November 1, 2016, http://lib.de.us/files/2015/01/Delaware-Libraries-White-Space-Pilot-Update-Jan-2015.pdf.

49. Knapp, “FCC Announces Public Testing of First Television White Spaces Database.”

50. See https://www.google.com/get/spectrumdatabase/.

51. Bertot et al., "Public access computing and Internet access in public libraries.”

52. Bertot et al., "The Impacts of Free Public Internet Access." See also Horrigan, "Libraries 2016."

53. Paul T. Jaeger, Lesley A. Langa, Charles R. McClure, and John Carlo Bertot, "The 2004 and 2005 Gulf Coast Hurricanes: Evolving Roles and Lessons Learned for Public Libraries in Disaster Preparedness and Community Services," Public Library Quarterly 25, 3/4, (2007), 199-214.

54. Ibid. 
55. Bertot et al., "Public access computing and Internet access in public libraries.”

56. Ibid.

57. Paul T. Jaeger, Ben Shneiderman, Kenneth R. Fleischmann , Jennifer Preece, Yan Qu, Philip Fei $\mathrm{Wu}$, “Community response grids: E-government, social networks, and effective emergency management," Telecommunications Policy 31 (2007): 592-604, https://doi.org/10.1016/j.telpol.2007.07.008.

58. Ibid., 595.

59. Laurie Putnam, "By choice or by chance: How the Internet is used to prepare for, manage, and share information about emergencies," First Monday 7, no.11 (2002), https://doi.org/10.5210/fm.v7i11.1007.

60. Ibid.

61. Ibid.

62. Jaeger et al., “Community response grids,” 598. Jaegar et al. describe how the Internet combines the best of one-to-one, one-to-many, many-to-one, and many-to-many in terms of the flow and quality of information. One-to-one communication is slow; many-to-one only benefits the central network, while outsiders reporting emergencies do not learn what others are reporting; one-to-many is inefficient, limited, and assumes the broadcaster has the appropriate information and can get it to those that need it most; many-to-many can create "information overload" of questionable content.

63. Ibid., 599.

64. Jaeger et al., “The Intersection of Public Policy and Public Access,” 3.

65. Cohron, "The Continuing Digital Divide in the United States," 84.

66. Kinney, "The Internet, Public Libraries, and the Digital Divide," 120.

67. Ibid., 148.

68. Jaeger et al., “Community response grids,” 599.

69. Bertot et al., "The Impacts of Free Public Internet Access,” 299. 